\title{
ESR2 Status
}

National Cancer Institute

\section{Source}

National Cancer Institute. ESR2 Status. NCI Thesaurus. Code C159152.

Refers to the presence or absence of ESR1 in a sample. 